\title{
Clinical Impact of Gait Training Enhanced with Visual Kinematic Biofeedback: Patients with Parkinson's Disease and Patients Stable Post Stroke
}

Nancy Byl ${ }^{1}$, Wenlong Zhang ${ }^{2}$, Sophia Coo $^{1}$, Masayoshi Tomizuka ${ }^{2}$

This work was supported by National Science Foundation under Grant CMMI-1013657. None of the authors have a conflict of interest to report.

1. N. Byl and S. Coo are with the Department of Physical Therapy and Rehabilitation Science, School of Medicine, University of California, San Francisco, CA 94158 USA (email: Byln@ptrehab.ucsf.edu; sophiacoo@gmail.com)

$\mathrm{N}$. Byl was responsible for recruiting subjects and gait training, training the research assistants for subject evaluation, data documentation and entry and S. Coo was responsible for data analysis.

2. W. Zhang and M. Tomizuka are with the Department of Mechanical Engineering, University of California, Berkeley, CA 94720 USA (email: wlzhang@berkeley.edu; tomizuka@me.berkeley.edu)

W. Zhang and M. Tomizuka were responsible for innovating and building the sensor system and software programming for kinematic data collection and analysis. 


\section{Clinical Impact of Gait Training Enhanced with Visual Kinematic Biofeedback: Patients with Parkinson's Disease and Patients Stable Post Stroke}

\section{Abstract (290)}

As the world's population ages, falls, physical inactivity, decreased attention and impairments in balance and gait arise as a consequence of decreased sensation, weakness, trauma and degenerative disease. Progressive balance and gait training can facilitate postural righting, safe ambulation and community participation. This small randomized clinical trial evaluated if visual and kinematic feedback provided during supervised gait training would interfere or enhance mobility, endurance, balance, strength and flexibility in older individuals greater than one year post stroke (12) or Parkinson's disease (PD) (12). Twenty-four individuals consented to participate. The participants were stratified by diagnosis and randomly assigned to a control (usual gait training [12]) or an experimental group (usual gait training plus kinematic feedback [12]). At baseline and 6 weeks post training (18 hours), subjects completed standardized tests (gait speed, endurance, balance, strength, range of motion). Gains were described overall, by treatment group and by diagnosis then compared for significance using nonparametric statistics. Twenty-three subjects completed the study with no adverse events. Across all subjects, by diagnosis (stroke and PD) and by training group (control and experimental), there were significant gains in mobility (gait speed, step length, endurance, and quality), balance (Berg Balance), range of motion and strength. There were no significant differences in the gain scores between the control and experimental groups. Subjects chronic post stroke made greater strength gains on the affected side than subjects with PD but otherwise there were no significant differences. In summary, during supervised gait training, dynamic visual kinematic feedback from wireless pressure and motion sensors had similar, positive effects as verbal, therapist feedback. A wireless kinematic feedback system could be used at home, to provide feedback and motivation for self correction of gait while simultaneously providing data to the therapist (at a distance).

Key words: gait training, stroke, PD, kinematic biofeedback, wireless 


\section{Introduction}

The world's population is aging. By 2030, nearly $20 \%$ of the world's population is projected to be over 65 years of age. (1) With aging, there are increased numbers of patients who not only become physically inactive, but are challenged with injurious falls, cardiovascular insults or neurodegenerative disease. (2-6) Exercise is critical for healthy aging and maintaining independence, community participation and quality of life for individuals with neurological impairments. $(7,8)$ Despite dopaminergic medications, older patients with Parkinson's disease (PD) commonly have difficulty maintaining community ambulation due to rigidity, bradykinesia, tremors, poor postural righting, decreased proprioception and dual tasking and freezing. (9-12) Further, while an increasing number of people survive a stroke, 6 months post insult, hypertonia, weakness, sensorimotor impairments, pathological synergies and sometimes compromised attention or cognition can lead to difficulties with safe, independent transfers and ambulation (15$19 \%$ ), difficulty with activities of daily living and limitations in community participation (13-49\%). (13-16) Research supports the benefits of task oriented, progressive gait training to improve mobility, balance, strength and flexibility post neurological impairment, but, unfortunately, the intervention strategies are not 100\% effective. (14, 18-22)

When designing evidence based gait re-training, therapists integrate information from the history (e.g. family, psychosocial, medical, mental and physical), the clinical examination (e.g. posture, balance, biomechanics, sensory, motor, motor planning and ambulatory skills) and previous treatment to identify impairments and dysfunction. Then, together, the physician, the patient, the family and the therapist outline and prioritize short and long term training goals, which are integral to defining a plan of care. (23) The next step is to identify the tasks which must be practiced to achieve the desired outcomes. (19) This is followed by defining the intensity of supervised therapy needed and establishing a home exercise program to integrate and transfer skills into activities of daily living, recreation, social interactions and work. (24-26) Sometimes therapists also recommend assistive, robotic or computerized devices to improve stability, enhance somatosensory 
information, move a limb or provide feedback about accuracy, kinematics or efficiency of movement. (27-28) These recommendations are matched to patient ability, interests, attention and concentration.

The precise biomechanical abnormalities in walking may not be accurately nor comprehensively assessed with visual analysis supplemented with standard measurements of endurance, gait speed, performance time, range of motion and strength. Kinematic gait data on sequencing, velocity, symmetry, and ground reaction forces could facilitate improved mobility training through the enrichment of knowledge of both the therapist and the patient. (29-33) The challenge is to determine if heightened, dynamic sensory feedback is disruptive or reinforcing for individuals with neurological impairments who are trying to improve gait efficiency (e.g. speed, quality, symmetry, stability, movement initiation) If visual kinematic feedback enriches verbal instructions from the therapist and the patient can integrate both verbal and visual information to correct gait impairments, then a wireless kinematic biofeedback system could be useful in the clinic with "one on one" supervision or possibly at home with remote data analysis and feedback.

The purpose of this study was to evaluate the effectiveness of "one on one" supervised gait training with and without visual, kinematic biofeedback to improve mobility, balance, strength and flexibility for patients with gait impairments post diagnosis of PD or chronic stroke. We hypothesized "one on one" progressive gait training with a therapist (18 hours) would be associated with improved mobility, balance, strength and flexibility. We also hypothesized that the improvements would be similar for "one on one" progressive gait training and "one on one" gait training supplemented with kinematic biofeedback. We also proposed that patients one year post stroke would make similar gains in mobility, balance, strength, flexibility and endurance following gait training (with or without kinematic feedback) compared to adults one year post diagnosis of PD. 


\section{Material and Methods}

\subsection{Subjects}

Adults, males or females, 30-75 years of age, who had gait impairments one year or more post stroke or diagnosis of PD (without other significant health problems) were eligible for study participation. The subjects were independent in self-care, able to communicate in English (or come with an interpreter), able to follow instructions, interested in being more mobile and could rise from a chair and walk without personal assistance for a minimum of 100 feet. All of the subjects had participated in previous research at the University of California, San Francisco (UCSF) or had received physical therapy in the UCSF Faculty Practice or the UCSF PT Heath and Wellness Center. Twenty six subjects were contacted by phone, met the eligibility screen and were scheduled for consent, testing to confirm eligibility (independence, depression, cognition, severity of PD and stroke) and baseline testing. Mental alertness was assessed with the VA mental Status Test ( $\geq 24 / 30$ ) (34) and the Beck Depression Scale (35) was administered to rule out individuals with severe depression (<12). To confirm independence at home, the Café 40Functional Independence Scale (36) was administered ( $\geq 50 \%)$. The severity of impairment post PD had to fall between I and III on the Hoehn and Yahr Scale (37) and subjects post stroke had to obtain a minimum score of 10 on the lower extremity FuglMeyer. (38) One subject did not meet the eligibility criteria and a second subject met the eligibility requirements but could not commit the time to gait training therapy twice a week. Thus, 24 subjects proceeded to the baseline evaluation. The subjects were then coded by diagnosis and randomly assigned to a control (usual gait training) or an experimental group (usual gait training reinforced with kinematic visual feedback). This study was approved by the Committee on Human Research at UCSF.

\subsection{Baseline and Follow up Evaluations}

At baseline and following 12 training sessions, the subjects were administered standardized tests for mobility, balance, strength and range of motion (ROM). The subjects were also asked to report the number of falls experienced the month before admitted and the month during training. Mobility (gait speed) was measured with the Timed 10-Meter Walk at fastest speed, (39-41) the Six Minute Walk, (42) the Dynamic 
Gait Index, (43) and the Tinetti Gait Assessment (Quality). (44) In order to comprehensively measure balance (45) three different tests were administered: Timed Up and Go Test (TUG) (46,47), Five Times Sit to Stand (FTSTS) Test $(48,49)$ and the Berg Balance Scale. (50-53) Strength was tested following the guidelines for manual muscle testing (54) using the Microfet Dynamometer. (55) The force generated for each muscle group (pounds) was summed to a total score of strength for each leg. Range of motion was measured with a goniometer following standard guidelines (56), with the degrees of motion measured at each joint in the lower extremity summed to create a total flexibility score for each leg. The subjects with PD also completed a self-report questionnaire on freezing. (57) and both the subjects post stroke and those with PD completed self-report questionnaires on sleep, (58) fatigue, (59) resilience, (60) and pain (Visual Analog ScaleVAS). (61)

\subsection{Equipment}

\subsubsection{Smart Shoes}

A pair of smart shoes was developed to measure the ground eon reaction forces (GRF) on the foot during ambulation. (30,33). Four pressure sensors were employed to measure the GRF on the toe, the first and second metatarsophalangeal joint (Meta12), the fourth and fifth metatarsophalangeal joint (Meta45), and the heel. Silicone tubes were wound into air bladders and barometric pressure sensors with an equivalent weight measurement range up to 200 lbs. Figure l shows the silicone tubes on the bottom of the shoe pad and other components of the smart shoes. The smart shoes were only worn by the subjects during the training sessions but not the evaluation. 


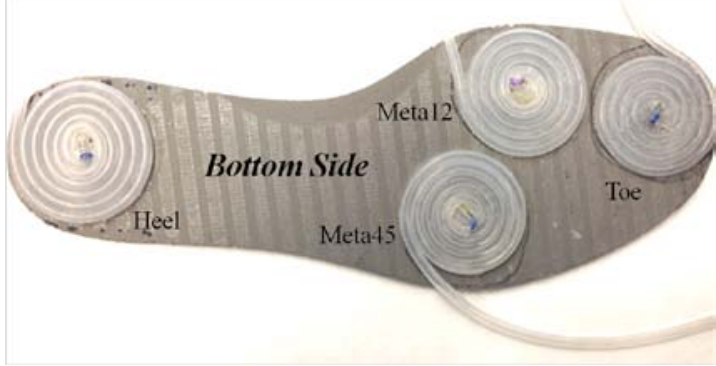

(a)

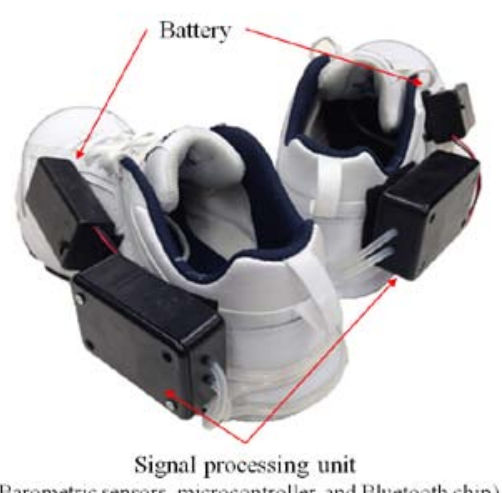

(b)

Figure 1: Smart shoes. (a) Bottom side of the shoe pad; (b) Components of a pair of smart shoes

The pressure sensor outputs were read by a microcontroller through analog input channels and the sensor signals were sent out through a Bluetooth module. (33) A 9V alkaline battery was used to power the whole system, and it could work consecutively for one and a half hours. The sampling rate of the smart shoes could go up to $50 \mathrm{~Hz}$ with the Bluetooth module. Based on the ground contact force distribution, one could quantitatively analyze the pattern of the walking and detect gait abnormalities.

\subsubsection{Wireless Joint Angle Sensors}

While engaged in training with the sensor system, the subjects wore the smart shoes. The sensor nodes were mounted on a pair of tight sport pants (to shorten the setup time) or velcro attached to the subject's pants. The wireless joint angle sensors were developed to measure joint rotations in three dimensions. (33) An inertial measurement unit (IMU) sensor stick was employed in the sensor. It included a three-degrees-of-freedom accelerometer, magnetometer and gyroscope. A microcontroller read the raw sensor data from the IMU sensor stick and a ZigBee module was employed to send out the raw sensory data wirelessly. The sensor node was powered by a two-cell Li-Po battery and it could work continuously for one hour. The dimension of the joint angle sensory node is 2.5 inches $\times 1.5$ inches $\times 1$ inch and its weight is approximately 0.2 pounds including the battery. Figure 2 shows the packing of a wireless joint angle sensor node and several sensor nodes attached to a pair of pants. 


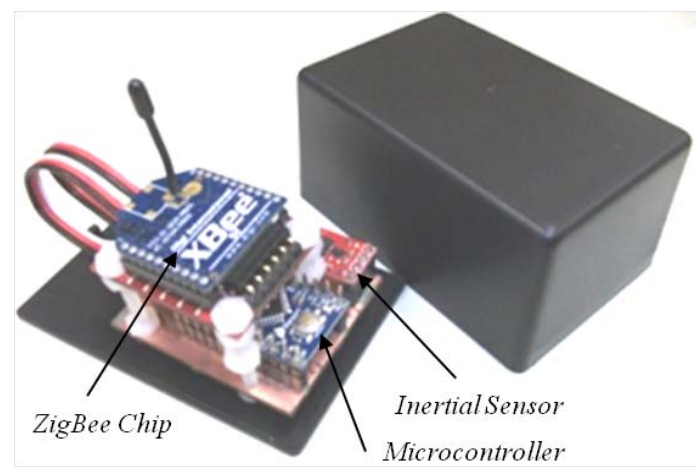

(a)

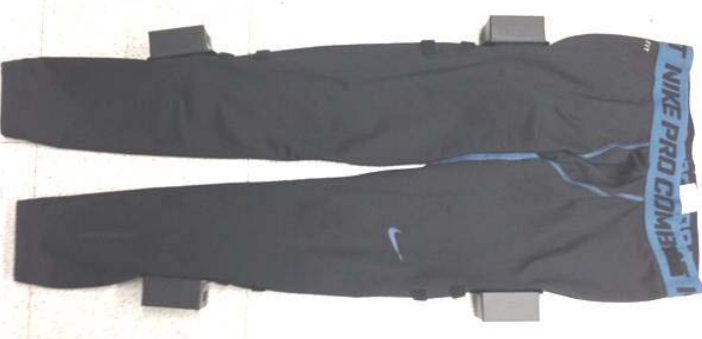

(b)

Figure 2: Wireless joint angle sensors. (a) a wireless joint angle senosr node; (b) joint angle sensor nodes attached to a pair of pants

The smart shoes and joint angle sensors sent measurements to a laptop wirelessly and a LabVIEW program was developed to read the sensor signals through serial ports. The LabVIEW program processed the raw data from the sensors, displayed sensory data on a user interface and sent selected data to an iPad for visual feedback through the Bluetooth Low Energy protocol. All the raw and processed data were stored in the laptop and could be used for future clinical studies. Figure 3 shows the user interface on an iPad, which displays foot pressing indicators, step lengths, stride widths, and toe-out angles. Pressure measurements from the smart shoes were displayed on the laptop. (Figure 4)

To measure joint rotations of hips, knees, and ankles on both sides, seven joint angle sensors were required. The sampling rate of the system can go up to $30 \mathrm{~Hz}$. This rate is sufficient considering the frequency of human walking is around $1 \mathrm{~Hz}$. The joint angle sensors were only worn by the subjects during the training sessions but not the evaluation. 


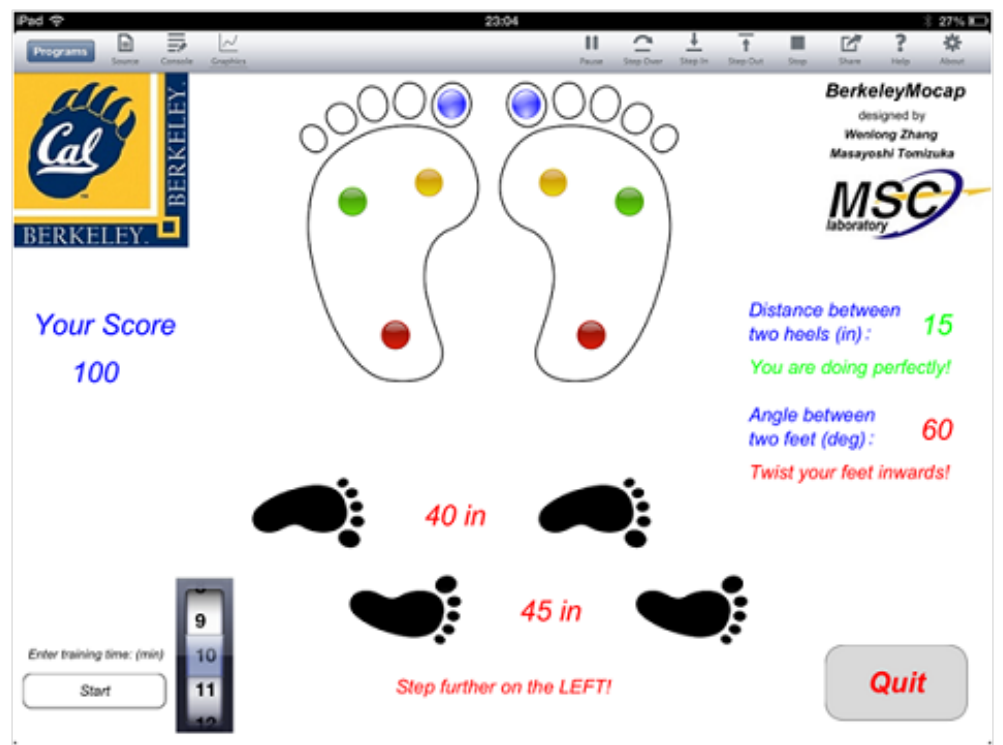

Figure 3: Information presented to patient: ground contact indicators and kinematics

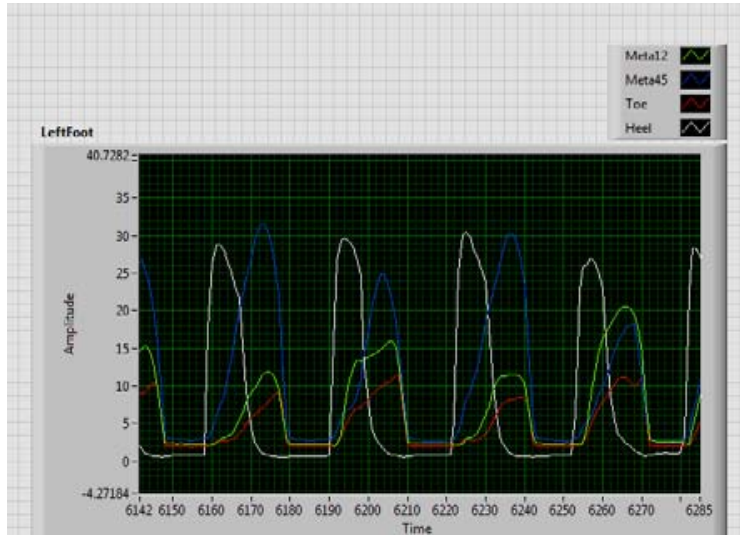

(a)

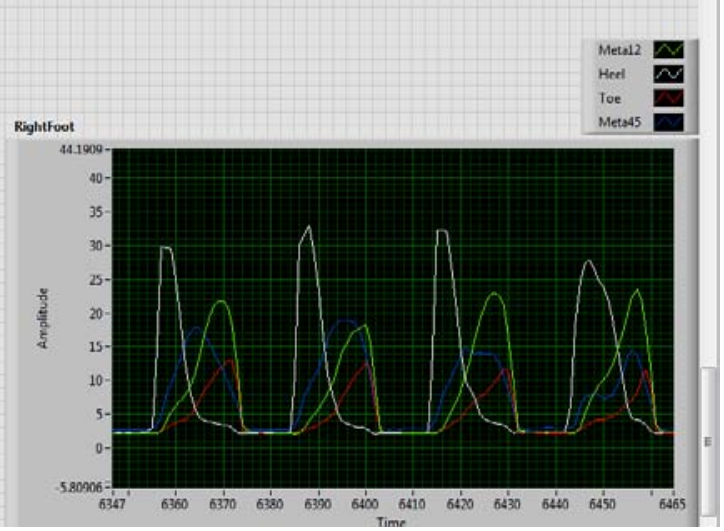

(b)

Figure 4: Feedback to patients on gait: timing, location and amplitude of ground contact forces. (a) affected side; (b) unaffected side. (White = heel; Blue = lateral border of mid-foot; Green = medial border mid-foot; Red = first toe). The patient put more force on the lateral border of the affected side, which led to leaning and fall.

\subsection{Gait Training}

Gait training was provided with "one on one” supervision by a physical therapist (PT) and a research assistant (RA) trained by the physical therapist. Both the PT and the RA provided supervised gait training in the same location at the same time, each with one subject. The training took place in the PT Health and Wellness Center. Weights, agility ladders, steps, obstacles, theraband, balls and treadmills were available for training. The gait training activities were progressive and task-oriented, following the principles of 
neural adaptation (62) and matched to the ability and objectives of each subject $(11,13,15,17,19,22,63,64)$. The gait training activities integrated exercises for good postural alignment, balance, strengthening of hip abduction, coordination stretching (heel cord, hip, and hamstrings) and dual tasking. Mobility training was done indoors and outdoors, over ground and on the treadmill. The gait training activities were similar for subjects in the control and experimental groups. (See examples of component task practice gait training activities in Table 1). The subjects in the experimental group wore the smart shoes and the pants for about 30 minutes during the gait training session. The subjects received the visual kinematic feedback on the computer screen interpreted by the engineer and the therapist.

Over 6-8 weeks, all subjects were scheduled for 12 visits (90 minutes each visit). The subjects were also encouraged to walk daily at home and continue usual participation in community activities (e.g. social commitments, individual and group exercise programs at home or at a fitness center).

\subsection{Study Design and Statistical Analysis}

This was a small, randomized clinical trial. Baseline, post exercise and gain scores (post exericise minus baseline test scores) for all dependent measures were described by mean, standard deviation, and effect size. Significance of gain scores was analyzed across all subjects, by control and experimental groups and by diagnostic groups using nonparametric statistics (Matched Pair Wilcoxon for exercise based change scores and Mann Whitney $U$ for group differences). $(65,66)$ Each analysis was tested at $\mathrm{p}<0.05$ (two tailed). Due to small numbers, within the stroke group and the PD group, differences between the control and experimental intervention groups were described, but not analyzed for significance.

\section{Results}

The mean age of all subjects was 63.5 years, with 16 males and 8 females. There were 12 subjects in the control group (5 with PD and 7 post stroke) and 12 in the experimental group (7 with PD and 5 with stroke). At baseline, there were no significant differences in 
the screening and descriptive variables for subjects assigned to the control or experimental group. (See Table 2) Of the 12 subjects post stroke, 5 had a right hemiplegia and 7 had a left hemiplegia. The baseline Fugl Meyer scores for the lower limb ranged from 5 to 23 .

All but one subject completed all 12 training sessions (one subject missed one session). Due to an unrelated medical illness, one subject in the experimental group (female, chronic post stroke) was unable to complete the study. No adverse events occurred during the study. Subjective signs and symptoms were monitored descriptively during training with no reports of exacerbation of signs and symptoms. The subjects reported low levels of pain at the beginning and the end of gait training (1.6-1.9 on the Visual Analog Scale). The subjects also reported minimal changes in fatigue, resilience, sleep, and freezing ( $\leq 7 \%)$ A month before the beginning of the study, subjects reported an average of 13.7 falls. During the month of gait training, the subjects reported an average of 7.3 falls. The majority of falls were experienced by patients with PD.

The kinematic feedback was provided to the subjects with the therapist present. Although not specifically studied, the kinematic feedback and the verbal feedback from the therapist was consistent. Across all subjects in the experimental group, the kinematic data indicated decreased foot clearance during the swing phase, delayed knee flexion at heel rise, inconsistent heel strike (frequently hitting with the forefoot), poor translation of the ground reaction force from the heel to the midfoot to the first ray, reduced time on the affected leg during single leg stance with a shorter step and decreased velocity on the affected side.

\subsection{Results across All subjects}

Post exercise, across all subjects, there were significant gains baseline in mobility (gait speed, step length, endurance, dynamic task responses during walking [DGI]), gait quality [Tinetti Gait Assessment]), balance (BBT), strength (affected and unaffected) and range of motion (affected and unaffected). There were moderate to large effect sizes, ranging from 0.37 to 2.0 with a mean of 0.81 ). (See Table 3 ) 


\subsection{Control versus Experimental Groups}

Table 4 summarizes the gain scores for the control and experimental groups. Both the control and experimental groups made significant gains on all of the dependent variables except the timed balance tests (FTSTS and TUG). In addition, the experimental gait training group did not make significant gains in ROM on the most affected side. Comparing the control and experimental groups, there were no significant differences in the gain scores for any of the dependent variables. The effect sizes were low with a mean effect size of 0.27 .

Table 5 summarizes the post exercise gain scores for the control and experimental groups within the PD and Stroke groups. Within the stroke group, the effect sizes were greater for the experimental group compared to the control group for the Tinetti Quality Inventory (1.24), the Dynamic Gait Inventory (DGI 0.43), FTSTS (-0.31), the Berg Balance Test (BBT; 0.42) and flexibility (0.30 to 0.56), with the effect sizes ranging from moderate to large. For the subjects with PD, the Experimental group experienced a greater effect size than the control group on the Dynamic Gait Inventory (DGI; 0.23), FTSTS (-0.19), TUG (-1.10), the Berg Balance Test (0.89) strength (0.30) and flexibility (0.56-0.76). The effect sizes ranged from small to large. Within both the stroke and PD diagnostic groups, the control group achieved larger effect sizes than the experimental group in mobility (10 meter walk, Six Minute Walk) and strength with effect sizes ranging from 0.30 to 1.24 .

\subsection{Results between Diagnostic Groups: Stroke and PD}

Post training, the subjects in the stroke group made significant gains on all of the dependent variables except endurance (6 Minute Walk) and timed balance (FTSTS and TUG). The PD group made significant gains on all of the dependent variables except for gait speed (10 meter walk) and timed integrated balance (TUG). For the subjects in the stroke group, the effect sizes for the significant differences ranged from a low of 0.28 to a high of 3.44 (strength, affected side). The effect sizes for the significant gains for the subjects in the PD Group ranged, from a low of 0.39 to a high of 2.14 (strength, unaffected side). See Table 6. Subjects post stroke made greater strength gains on the 
affected side compared to subjects with PD (effect size of the difference score 1.84) However, there were no other significant differences in the gain scores for subjects post stroke compared to those with PD. (See Table 6).

\section{Discussion and Conclusions}

In this small randomized clinical trial, 6 weeks of progressive gait training, with or without kinematic feedback, significantly reduced the number of falls and enhanced mobility, endurance, strength and flexibility. Supplementing visual kinematic feedback data with supervised gait training (experimental group) did not disrupt gait training efficiency or effectiveness as confirmed by similar gains for the subjects in the control and the experimental groups. By diagnosis, subjects post stroke made greater strength gains on the affected side compared to subjects with PD, but otherwise there were no significant differences in the clinical outcomes.

Across all subjects, the gains in gait speed met the criteria for a minimal clinically important difference (MCD). (67) However, when stratified by diagnosis, only the subjects in the stroke group met the MCD level of improvement in gait speed. Compared to the age and gender related norms for fast walking by community dwelling elderly individuals, (68) the average fast walking speed in this study for subjects post stroke was slower than the norms $(1.3-1.4 \mathrm{~m} / \mathrm{sec}$ compared to the norm of $1.87-2.05 \mathrm{~m} / \mathrm{sec})$. The gains in distance walked in six minutes did not meet the MCD (16 meters compared 70 meters) with the distance walked lower than healthy age matched community dwelling elders (266-283 meters compared to the norm of 409-521 meters). (68) Part of the differences in endurance testing in this current study related not only to the slowness of walking but in the setting where the assessment took place, subjects had to turn each 10 meters instead of each 30 meters as recommended for standard administration of the Six Minute Walk. The average scores on the Berg Balance Test (41-56) placed subjects in the category for "low fall risk".(50) However, on the TUG and the FTSTS, the subjects did not meet the minimal, clinically significant gains in performance (2.5-3.0 seconds) (47) The subjects in this study performed the TUG (17.4 -18.4 seconds) less efficiently than age matched community dwelling non fallers (8.4 sec [1.7]) (69) but slightly better that 
elderly patients with vestibular disorders (47) or older community fallers (22.2 sec [9.3]).(69) Based on performance on the TUG and the FTSTS, the subjects would be categorized as "high risk" for falls. (69) Research suggests the Berg Balance Test is relatively good for identifying the non-fallers, but not necessarily sensitive to identifying the fallers. (52) In this study, half of the subjects reported no falls the month before participating in the study, while 3 subjects were falling over 100 times a month. At the end of gait training, 18 of the 23 subjects reported they did not experience any falls during the month of training.

This study was designed to study the effectiveness of gait training to improve mobility, balance, strength and flexibility in patients chronic post stroke or PD as well as to evaluate the safety, efficiency and consistency of dynamic visual kinematic feedback when added to "one on one” gait training. While visual kinematic enhancement is consistent with the principles of behavioral training, the investigators were concerned the visual feedback would disrupt performance and concentration in patients with neurological impairments. Theoretically, wireless, dynamic, visual, kinematic feedback could: 1) provide real time quantitative, gait information to enhance the knowledge base of the patient and the physical therapist; 2) enable the evaluation of consistency between the objective visual kinematic analysis and the clinical assessment that guides the verbal feedback from a therapist; 3) enable therapists to use objective kinematic data to document and elaborate on the clinical gait analysis to improve the foundation for designing a plan of care; 4) create an objective measure for documenting changes in gait over time; 5) establish a system for giving feedback to patients during home training to correct gait impairments when a therapist was not present; and 6) provide a method of remotely transmitting data to the therapist regarding compliance with home training, persistence of gait abnormalities and need for modification of the exercise program. (3033)

This study confirmed subjects in a chronic state post stroke or with mild to moderate PD could safely receive visual kinematic information without disrupting gait. However the kinematic information was provided while the subject was standing or sitting after 
performing a few walking trials. It was not provided to the subject during the walk. Further, the subjects did not want to wear the iPad around their waist while walking.

Across diagnosis, there were no significant differences in the gain scores on the dependent variables between subjects in the experimental group compared to the control group. However, within diagnostic group, some interesting trends were noted between the control and experimental groups. For example, subjects post stroke in the experimental group, achieved a larger gain and effect size than the control group on two of the gait measurements (the Tinetti Gait Assessment and the Dynamic Gait Inventory), two of the balance tests (FTSTS and the Berg Balance Test) and flexibility. Within the subjects with $\mathrm{PD}$, those assigned to the experimental group achieved a larger effect size on the Dynamic Gait Inventory and two of the balance tests (FTSTS and TUG). Within each of the diagnostic groups, the subjects in the control group achieved larger gains in gait speed, step length and strength. It is possible the detailed information about the movement of the center of gravity and the sequence of the ground reaction forces, along with the asymmetries of step length, single limb support and limb velocity improved balance responses and the ability to do dual tasking while walking (as required in the DGI). However, the subjects in the experimental group may have been so focused on improving gait mechanics that they walked less quickly than subjects receiving only verbal feedback from the therapist.

The next step is to make the human motion monitoring system easier to use at home. When this is accomplished, it will be possible to test the hypothesis that patients post stroke or with PD could improve gait and balance by using visual kinematic feedback at home without "one on one” supervision. Further, it will be possible to test the hypothesis that visual kinematic feedback supplemented with remote transmission of performance information to a therapist would allow the therapist to modify the gait training plan and increase gains in gait, balance, strength and flexibility compared to kinematic biofeedback alone. The visual feedback could provide the support elders may need (74) to stay compliant with their exercise programs and life style modifications. The visual kinematic feedback may enable elders to maintain sufficient gait speed and stability 
required for independence in self care and community participation. (75) Further, studies will be needed to train therapists how to remotely analyze data from wireless technology to provide timely and appropriate upgrades to the patient's exercise program. It will also be important to show that visual kinematic feedback supplemented with on line feedback from a therapist will be equally as effective as expensive "one on one" feedback. Finally, it will be critical to create a mechanism within the health care system to cover the expenses associated with rehabilitation technology and the provider of tele-healthcare services.

There were some limitations in this small, randomized clinical trial. The number of subjects was small, particularly within each diagnostic group and within the control and experimental groups. The small numbers could have limited the ability to find differences between the control and the experimental groups. On the other hand, the effect sizes were low where no significant differences were found and moderate to high where statistically significant differences were reported. In this small, preliminary randomized trial, the gain scores for each dependent were tested for significance at $p<0.05$. This type of analysis assumes independence of each of the outcome measurements. Although each dependent variable was measured independently, it is likely that speed, endurance and step length, for example, were not completely independent but interacted in the measurement of mobility. Similarly there was probably some shared variance in the performance of balance as measured by three different balance tests. Multiple tests characterizing a common parameter can increase the risk of a type-II error. $(65,66)$ The activities for the "one on one" intervention were selected from a list of common tasks relative to balance and walking. These activities may have not have been perfectly matched to the patient's priority lists (19) (e.g. one patient wanted to learn to jog over ground, but the therapist did not think the patient was safe to embark on this task). Intervention ranged from 5-7 weeks to accommodate subject schedules. This may not have been sufficient time or sufficient intensity to measure differences in outcomes between the two training groups. (72) On the other hand, while more intensive therapy may be associated with greater gains, there is evidence that measurable effects can be achieved in 4-6 weeks. $(73,74)$ The engineer provided verbal and presented visual, 
kinematic feedback to the patient after selected walking trials to enhance the feedback generated during walking. Although a belt support system was available to support the iPad, it was clumsy to use and difficult to observe while walking. The Smart Shoes were classified as small, medium and large. Consequently the shoes were not a perfect fit for the subjects. However, the subjects did not wear the Smart Shoes or the sensors for baseline and follow up testing. Further, at this stage of development, the subjects needed assistance to put on the smart shoes and pants as well as link the sensors to the iPad. For patients post stroke, it will be important for subjects to be able to put on the human motion monitoring system with one hand. The subjects participating in this study represented only individuals with a stroke or with PD. In future research studies, it will be important to include healthy elders as well as individuals with a variety of different types of injuries or degenerative conditions.

In conclusion, progressive, "one on one" task specific gait training can improve the efficiency and effectiveness of mobility, balance, strength and flexibility in patients in the chronic phase post diagnosis of PD or stroke. Wireless, dynamic kinematic, visual biofeedback can be provided within supervised "one on one" training sessions without disrupting attention to gait training activities. When this wireless human motion monitoring system is modified for simple donning and computerized connections, the visual-kinematic biofeedback system could enable patients to train more effectively at home while keeping the therapist abreast of gait training compliance, falls and persistence of gait abnormalities with the potential to analyze the kinematic feedback and schedule a telephone call, a computer interaction (e.g. Skype) or a clinic follow up visit to modify the home exercise program. 


\section{References}

[1] World population ageing: 1950-2050. http://www.un.org/esa/population/publications/ worldageing19502050.

[2] Hafner K., A tiny stumble, a life upended. The New York Times, Nov 3, 2014 http://nyti.ms/1A76mll.

[3] Statistics on Parkinson’s. http://www.pdf.org/en/parkinson's_statistics.

[4] Dorsey E.R., et al., Projected number of people with Parkinson's disease in the most populous nations: 2005-2030. Neurology 2007; 68 (5):384-86.

[5] Nussbaum, R.I., Ellis, C.E., Alzheimer's disease and Parkinson's disease. $N$ Eng $J$ of Med 2003; 348:1356-1364.

[6] Go A.S., et al., Executive Summary: Heart disease and stroke statistics-2014 update: a report from the American Heart Association. Circulation 2014; 129:399-410.

[7] Ahlskog J.E., Geda Y.E., Graff-Radford N.R., and Peterson R.C., Physical treatment as a preventive or disease modifying treatment of dementia and brain aging. Mayo Clin Proc 2011; 866:871-884.

[8] Fletcher, G.F., et al., Statement on exercise: benefits and recommendations for physical activity programs for all Americans. Circulation 1996; 94:857-862.

[9] Suchowersky O., Reich S., Perlmutter J., Zesiewicz T., Gronseth G., Weiner W.J., Practice parameters: diagnosis and prognosis of new onset Parkinson disease (an evidence-based review: report of the Quality Standards Subcommittee of the American Academy of Neurology). Neurology 2006; 66 (7):968-975.

[10] Allen N.E., Canning C.G., Sherrington C., Lord S.R., Latt M.D., Close J.C., et.al. The effects of an exercise program on fall risk factors in people with Parkinson's disease: a randomized controlled trial. Mov Disord 2010; 15 (9):1217-1225.

[11] Hirsch, MA, Farley, BG Exercise and neuroplasticity in persons living with Parkinson’s Disease. Eur J Phys Rehabil Med 2009; 45: 215-219.

[12] Gobbi L.T., Oliveira-Ferreiro M.D., Caetano J.D., Lirani-Silva E., Barbieri F.A., Stella F., et.al., Exercise programs improve mobility and balance in people with Parkinson’s disease. Parkinsonism Relat Disord, 2009; Suppl 3:S49-52. 
[13] Jorgensen H., et al. Recovery of walking function in stroke patients: the Copenhagen Stroke Study, Arch Phys Med Rehabil, 1995; 76:27-32.

[14] Kwakkel G., et al. Predicting disability in stroke-a critical review of the literature. Age and Ageing 1996; 25:479-489.

[15] Lang C.E., et al. The brain recovery core: building a system of organized stroke rehabilitation across the continuum of care. J Neurologic Physical Therapy 2011; 35:1-8. [16] Veerbeek J.M., et al. What is the evidence for physical therapy poststroke? A systematic review and meta-analysis. PLoS One 2014; 9(2):879-887.

[17] Boyd L.A., et al. Learning implicit effects of task and severity after stroke. Neurorehabil Neural Repair 2007; 21 (5):444-454

[18] Duncan P.W., et al. Management of adult stroke rehabilitation care: a clinical practice guideline. Stroke 2005; 36 (9):e100-43.

[19] Winstein C., et al. Infusing motor learning research into neurorehabilitation practice: a historical perspective with case exemplar from the accelerated skill acquisition program. J Neurol Phys Ther. 2014; May 13. [Epub ahead of print]

[20] Goodwin V.A., Richards S.H., Taylor R.S., Taylor A.H., Campbell J.L., The effectiveness of exercise interventions for people with Parkinson's disease: a systematic review and meta-analysis. Mov Disord 2008; 23 (5):631-640.

[21] Keus S.H., Munneke M., Nijkrake M.J., Kwakkel G., Bloem R., Physical therapy in Parkinson’s disease: evolution and future challenges. Mov Disord 2008; 24 (1):1-14.

[22] King L., Horak F.B., Delaying mobility disability in people with Parkinson's disease using a sensorimotor agility exercise program. Phys Ther 2009; 89 (4):1-10.

[23] Umphred D.A., et al. Neurological rehabilitation. 6th edition. St. Louis: Mosby, 2013.

[24] Lohse K.R., Lang C.E., Boyd L.A., Is more better? Using metadata to explore doseresponse relationships in stroke rehabilitation. Stroke. 2014; 45:2053-2058.

[25] Schaefer S.Y., Lang C.E., Using dual tasks to test immediate transfer of training between naturalistic movements: a proof-of-principle study, Journal of Motor Behavior 2012; 44(5):313-327. 
[26] Schaefer S.Y., Patterson C.B., Lang C.E., Transfer of training between distinct motor tasks after stroke: implications for task-specific approaches to upper-extremity. Neurorehabilitation 2013; 27(7):602-612.

[27] Chang W.H., Kim Y., Robot-assisted therapy in stroke rehabilitation. J of Stroke 2013; 15 (3):174-181

[28] Byl N.N., Mobility training using a bionic knee orthosis in patients in a post-stroke chronic state: a case series. J Med Case Rep 2012; 6(1):216.

[29] Liu, T., Inoue, Y., Shibata, K., Shiojima, K., A mobile force plate and threedimensional motion analysis system for three-dimensional gait assessment. IEEE Sensors J, 2012; 12(5):1461-1467.

[30] Kong, K., Tomizuka, M., A gait monitoring system based on air pressure sensors embedded in a shoe. IEEE/ASME Trans. on Mechatronics 2009; 14(3):358-370.

[31] Bae, J., Zhang, W., Tomizuka, M., Network-based rehabilitation system for improved mobility and tele-rehabilitation. IEEE Trans. on Control Systems Technology 2013; 21(5):1980-1987.

[32] Zhang, W., Zhu, X., Han, S., Byl, N.N., Mok, A. K., Tomizuka, M., Design of a network-based mobile gait rehabilitation system. In Proc. of IEEE Int. Conf. on Robotics and Biomimetics (ROBIO) 2012; 1773-1778.

[33] Zhang, W. Tomizuka, M., Byl, N.N., A wireless human motion monitoring system based on joint angle sensors and smart shoes. in Proc. of 2014 ASME Dynamic Systems and Control Conf. (DSCC) 2014; DSCC2014-5976, pp. 1-10, 2014.

[34] Tariq, S.H., Tumosa, N., Chibnall, J.T., Perry, H.M., Morley, J.E., The Saint Louis University Mental Status (SLUMS) examination for detecting mild cognitive impairment and dementia is more sensitive than the mini mental status examination (MMSE)-a pilot study. J Am Geriatri Psych 2006; 14 (11):900-910.

[35] Beck, A.T., Beck depression scale. The Psychological Corporation, Harcourt Brace and Company, San Antonio, 1996.

[36] Fung, S., Byl, N., Melnick, M., Callahan, P., Selinger, A., Ishii, K., Devins, J., Fischer, P., Torburn, L., Andrade, C.K., Functional outcomes: the development of a new instrument to monitor the effectiveness. Eur J Phys Med Rehabi 1997; 7:31-41. 
[37] Goetz C.G., Poewe W., Rascol O., Sampaio C.,Stebbins G.T., Counsell C., Giladi N., Holloway R.G., Moore C.G., Wenning G.K., Yahr M.D., Seidl L., Movement Disorder Society Task Force Report on the Hoehn and Yahr Staging Scale: Mov Dis 2004; 19 (9):1020-1028.

[38] Fugl-Meyer A.R., Jaasko L., Leyman I., Olsson S., Steglind S. The post-stroke hemiplegic patient. 1. a method for evaluation of physical performance. Scand J Rehabil Med 1975; 7:13-31.

[39] Bohannon W., Comfortable and maximum walking speed of adults aged 20-79 years: reference values and determinants. Age and Ageing 1997; 26: 15-19.

[40] Bohannon R.W., Andrews A.W., Normal walking speed: a descriptive metaanalysis. Physiotherapy 2011; 97:182-189.

[41] 10 meter walk test. http://www.rehabmeasures.org.

[42] American Thoracic Society ATS Statement: guidelines for the six-minute walk test. Am J Respir Crit Care Med. 2002; 166(1):111-117.

[43] Whitney S.L., Hudak M.T., Marchetti G.F., The dynamic gait index relates to selfreported fall history in individuals with vestibular dysfunction. J Vestib Res 2000; 10:99105.

[44] Tinetti, M.E., Performance-oriented assessment problems in elderly patients. J Am Geriatrics Society 1986; 34L:118-126.

[45] Foreman K.B,, Addison O., Kim H.S., Dibble L.E. Testing balance and fall risk in persons with Parkinson disease, an argument for ecologically valid testing. Parkinsonism and Related Disorders; 2011; 27:166-171.

[46] Podsiadlo D., Richardson S, The timed "Up and Go": a test of basic functional mobility for frail elderly persons. J of Am Ger Soc 1991; 39:142-148.

[47] Thrane G., Joakimsen R.M., Thornquist E., The association between timed up and go test and history of falls: the Tromse study. BNC Geriatrics 2007; 7:1-7.

[48] Meretta, B.M., Whitney, S.L., Marchetti, G.F., Sparto, P.J., Muirhead, R.J., The five times sit to stand test: responsiveness to change and concurrent validity in adults undergoing vestibular rehabilitation. J of Vestib Res 2006; 16:233-243.

[49] Bohannon RW. Reference values for the Five Times Sit to Stand Test: a descriptive meta analysis of data from elders. Percept Mot Skills 2006; 103(1):215-222. 
[50] Steffen, T.M., Hacker, T.A., Mollinger L., Age and gender-related test performance in community-dwelling elderly people: Six-Minute Walk Test, Berg Balance Scale, Timed Up and Go Test and gait speeds. Physical therapy 2002; 82:128-137.

[51] Berg K.O., Maki B.E. Clinical and laboratory measures of postural balance in an elderly population. Arch Phys Med Rehabil 1992; 73(11): 1073-1080.

[52] Berg, K.,Wood-Dauphinee, S.,et al. The Balance Scale: reliability assessment with elderly residents and patients with an acute stroke. Scand J Rehabil Med 1995; 27 (1):27-36. [53] Berg, K., Wood-Dauphinee, S., et al. Measuring balance in the elderly: validation of an instrument." Can J Public Health 1992; 83 Suppl 2:S7-11.

[54] Kendall F., McCreary E., Provance P., Muscles: testing and function. 5th ed. Williams \& Wilkins; 2005.

[55] microFET2 dynamometer. Hoggan Health Industries, Medical Products Division.

[56] Norkin C., White J., Measurement of joint motion: a guide to goniometry. 2nd ed. F. A. Davis; 1995.

[57] Giladi N., Tal J., Azulay T., Rascol O., Brooks, D.J., Melamed E., Oertel W., Poewe W.H., Stocchi F., Tolosa E., Validation of the freezing of gait questionnaire in patients with Parkinson’s disease. Mov Dis 2009; 24(5):655-661.

[58] Chaudhuri K., Pal S., DiMarco A., Whately-Smith C., Bridgman K., Mathew R., Pezzela F., Forbes A., Hogl B., Trenkwalder C., The Parkinson's disease sleep scale: a new instrument for assessing sleep and nocturnal disability in Parkinson's disease. $J$ Neurol, Neurosurg, and Psych 2002; 73(6):629-635.

[59] Neuberger G.B., Measures of fatigue: the fatigue questionnaire, fatigue severity scale, multidimensional assessment of fatigue scale, and short form-36 vitality (energy/fatigue) subscale of the short form health survey. Arthritis Care \& Research 2003; 49(S5):S175-S183.

[60] Wagnild G.M., Young H.M., Development and psychometric evaluation of the resilience scale. J Nursing Measurement 1993; 1(2):165-178.

[61] Price DD, McGrath PA, Rafii IA, Buckingham B. The validation of the Visual Analogue Scales as Ratio Scale Measures for chronic and experimental pain. Pain 1983; 17:45-56. 
[62] Merzenich, M., Soft-wired: how the new science of brain plasticity can change your life. Parnassus Publishing LLC; 2013.

[63] Boyd L.A., Vidoni E.D., Wessel B.D., Motor learning after stroke: is skill acquisition a prerequisite for contralesional neuroplastic change? Neurosci Lett. 2010; 482(1):21-25.

[64] Platz T., Impairment-oriented training (IOT) - scientific concept and evidence-based treatment strategies. Restorative Neurology and Neuroscience 2004; 22:301-315.

[65] Marascuilo LA, McSweeny M., Nonparametric and distribution-free methods for the social sciences. Brooks/Cole Publishing Company; 1977.

[66] Sackett D., Straus S., Richardson W., Rosenberg W., Haynes R., Evidence-based medicine second ed., Elsevier Churchill Livingstone; 2000.

[67] Subashan P.S., Mody S.H., Woodman R.C., Studenski S.A., Meaningful change and responsiveness in common physical performance measures in older adults. J Amer Ger Soc. 2006; 54 (5):743-749.

[68] Redelmeier D.A., Bayoumi A.M., Goldstein R.S., Guyatt G.H. Interpreting small differences in functional status: The Six Minute Walk Test in chronic lung disease patients. Am J Respir Crit Care Med. 1997; 155 (4): 1278-1282.

[69] Cook A.S., Brauer S., Woollacott M. Predicting the probability for falls in community-dwelling older adults using the Timed Up and Go Test. Phys Ther. 2000; 80:896-903

[70] Lo A., et al. Robot-assisted therapy for long-term upper-limb impairment after stroke. $N$ Engl J Med. 2010; 362(19):1772-1783.

[71] Byl N.N., Abrams, G.M., Pitsch E., Fedulow I., Kim H., Simkin M., Nagarajan S., Rosen J., Chronic stroke survivors achieve comparable outcomes following virtual task specific repetitive training guided by a wearable robotic orthosis (UL-EXO7) and actual task specific repetitive training guided by a physical therapist. J Hand Ther. 2013; 26(4):343-352.

[72] Kwakkel G., et al. Effects of augmented exercise therapy time after stroke: a metaanalysis. Stroke. 2004; 35:2529-2539.

[73] Ornish, D., Lin, J., Chan, J., Epel, E., Kemp C., Weidner, G., Marlin, R., Frenda S.J., Magbanua, J.M., Daubenmier, J., Estay I., Hills, N.K., Chainani-Wu, N., Carroll, P.R., 
Blackburn, E.H., Effect of comprehensive lifestyle changes on telomerase activity and telomere length in men with biopsy-proven low-risk prostate cancer: 5-year follow-up of a descriptive pilot study. Lancet Oncol. 2013; 14:1112-1120.

[74] Bowden M.G., Balasubramanian C.K., Behrman A.L., Kautz S.A., Validation of a speed-based classification system using quantitative measures of walking performance poststroke. Neurorehab and Neural Repair 2008; 22:672-675.

[75] Byl N., Roderick J., Mohamed O., Hanny M., Kotler J., Smith A., Tang M., Abrams G., Effectiveness of sensory and motor rehabilitation of the upper limb following the principles of neuroplasticity: Patients stable post stroke. Neurorehab and Neural Repair 2003; 17 (3):176-191

[76] Byl N., Pitsch E., Abrams G., Functional outcomes can vary by dose: learning-based sensorimotor traning for patient stable Post stroke Neurorehab and Neural Repair 2008; 22(5):494-504 